\title{
Performance Evaluation of Downlink Coordinated Multipoint Joint Transmission under Heavy IoT Traffic Load
}

\author{
Alaa M. Mukhtar $\mathbb{D}^{1},{ }^{1}$ Rashid A. Saeed $\mathbb{D}^{2},{ }^{2}$ Rania A. Mokhtar $\mathbb{D}^{2},{ }^{2}$ Elmustafa Sayed Ali $(\mathbb{D})^{3}$ \\ and Hesham Alhumyani ${ }^{2}$ \\ ${ }^{1}$ Department of Electronic Engineering, Sudan University of Science and Technology, Khartoum, Sudan \\ ${ }^{2}$ Department of Computer Engineering, College of Computers and Information Technology, Taif University, P.O. Box 11099, \\ Taif 21944, Saudi Arabia \\ ${ }^{3}$ Department of Electrical and Electronic Engineering, Red Sea University, Port Sudan, Sudan
}

Correspondence should be addressed to Elmustafa Sayed Ali; elmustafasayed@gmail.com

Received 7 September 2021; Accepted 10 December 2021; Published 6 January 2022

Academic Editor: Abdul Basit

Copyright (c) 2022 Alaa M. Mukhtar et al. This is an open access article distributed under the Creative Commons Attribution License, which permits unrestricted use, distribution, and reproduction in any medium, provided the original work is properly cited.

\begin{abstract}
Emerging 5G network cellular promotes key empowering techniques for pervasive IoT. Evolving 5G-IoT scenarios and basic services like reality augmented, high dense streaming of videos, unmanned vehicles, e-health, and intelligent environments services have a pervasive existence now. These services generate heavy loads and need high capacity, bandwidth, data rate, throughput, and low latency. Taking all these requirements into consideration, internet of things (IoT) networks have provided global transformation in the context of big data innovation and bring many problematic issues in terms of uplink and downlink (DL) connectivity and traffic load. These comprise coordinated multipoint processing (CoMP), carriers' aggregation (CA), joint transmissions (JTs), massive multi-inputs multi-outputs (MIMO), machine-type communications, centralized radios access networks (CRAN), and many others. CoMP is one of the most significant technical enhancements added to release 11 that can be implemented in heterogonous networks implementation approaches and the homogenous networks' topologies. However, in a massive 5G-IoT device scenario with heavy traffic load, most cell edge IoT users are severely suffering from intercell interference (ICI), where the users have poor signal, lower data rates, and limited QoS. This work is aimed at addressing this problematic issue by proposing two types of DL-JT-CoMP techniques in 5G-IoT that are compliant with release 18. Downlink JT-CoMP with two homogeneous network CoMP deployment scenarios is considered and evaluated. The scenarios used are IoT intrasite and intersite CoMP, which performance evaluated using downlink system-level simulator for long-term evolution-advanced (LTE-A) and 5G. Numerical simulation scenarios were results under high dense scenario-with IoT heavy traffic load which shows that intersite CoMP has better empirical cumulative distribution function (ECDF) of average UE throughput than intrasite CoMP approximately 4\%, inter-site CoMP has better ECDF of average user entity (UE) spectral efficiency than intrasite CoMP almost 10\%, and intersite CoMP has approximately same ECDF of average signal interference noise ratio (SINR) as intrasite CoMP and intersite CoMP has better fairness index than intrasite CoMP by $5 \%$. The fairness index decreases when the users' number increase since the competition among users is higher.
\end{abstract}

\section{Introduction}

The third-generation partnership projects (3GPPs) present a new system to increase the data rate and enhance the throughput, which is enhanced the LTE-A [1]. 3GPP added new features on 3GPP release 15 to develop Non-StandAlone (NSA) with $5 \mathrm{G}$ new radio (NRs) specification to pro- vide better user experience and spectral efficiency specifically at the cell edge and higher capacities scenarios [2]. Some of these features that introduced in 5G are to provide massive internet of things (IoT) users, multimode priority services, vehicles-to-everything (V2X), satellite 5G accesses, and service-based architecture (SBA), which were required to improve the architecture of CoMP, sophisticated CA, 


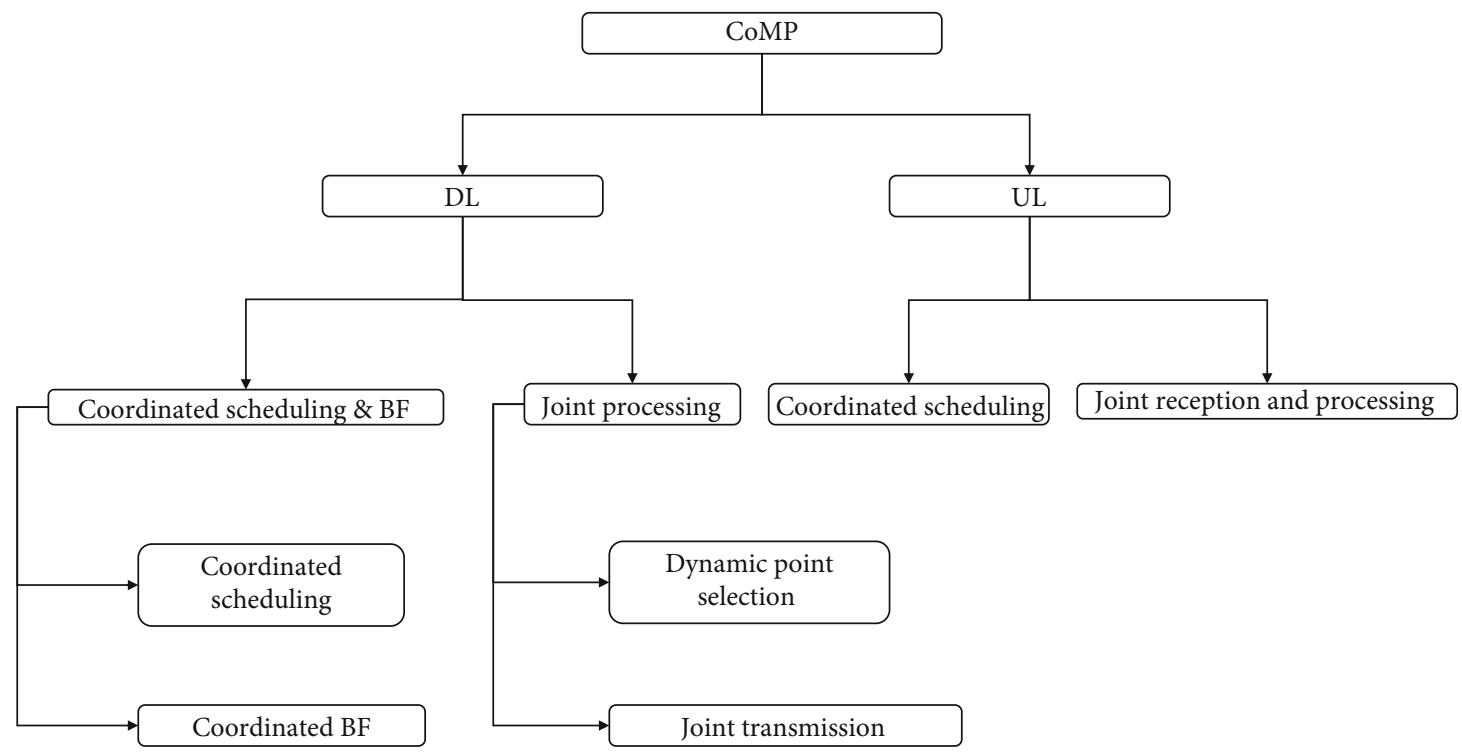

FIgure 1: The CoMP categorizes based on DL and UL.

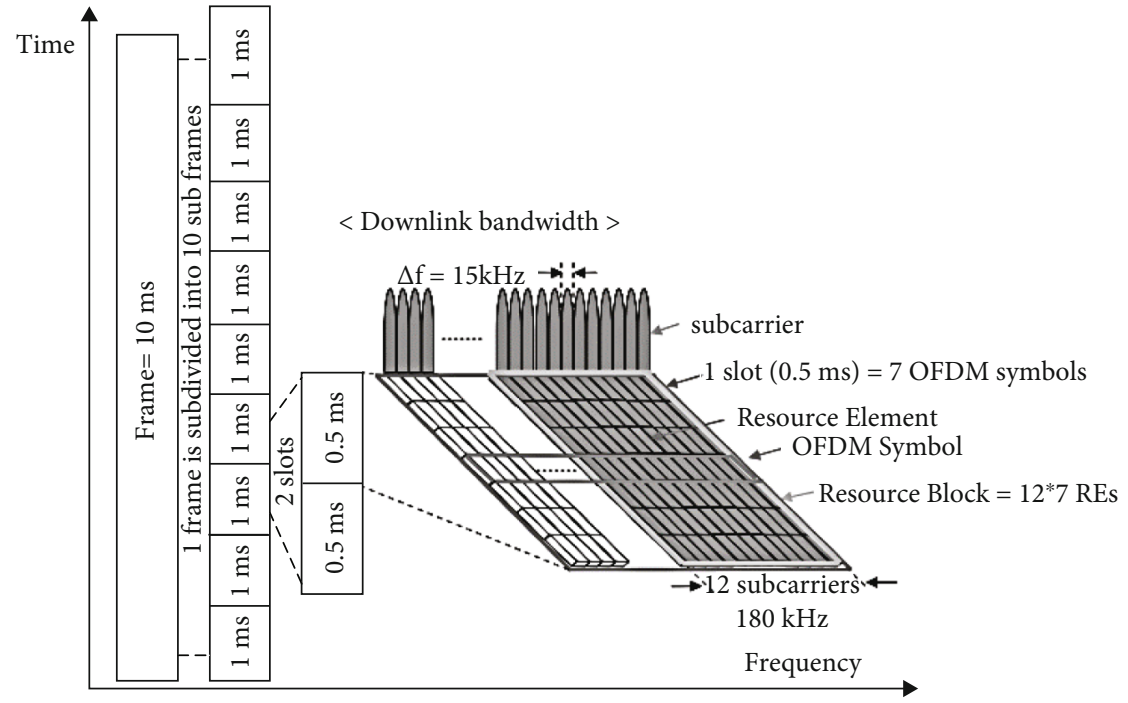

Figure 2: Downlink of 5G frame and radio resources.

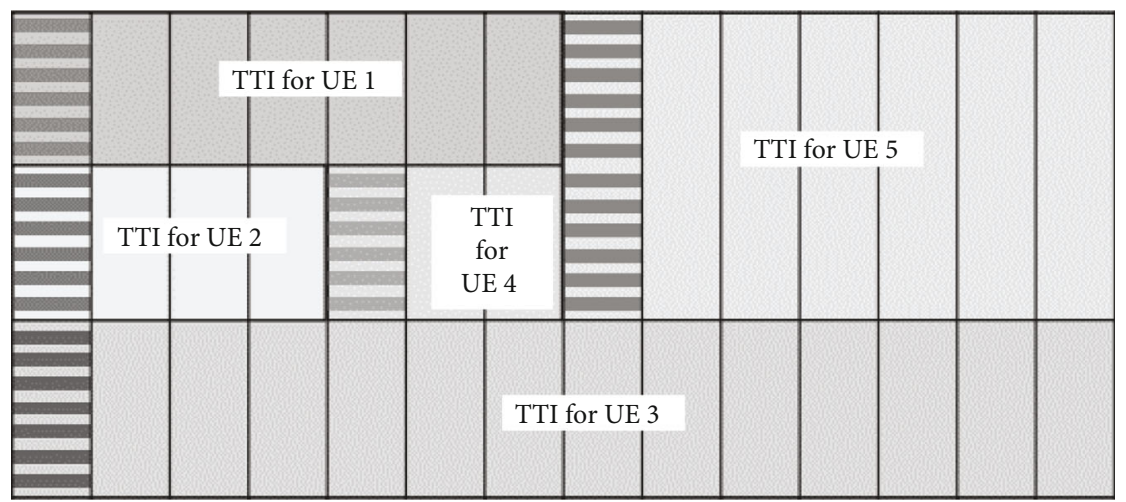

FIgURE 3: The 5G channels with various TTI-durations. 
TABLE 1: Summarized related work features and advantages.

\begin{tabular}{|c|c|c|c|}
\hline Citations & Purpose & Application feature & Advantage \\
\hline $\begin{array}{l}\text { Ouyang } \\
\text { et al. [13] }\end{array}$ & CoMP in LTE-advanced & $\begin{array}{l}\text { Cell-edge user experience in homogeneous and } \\
\text { heterogeneous networks }\end{array}$ & $\begin{array}{l}\text { Enhance the throughput in downlink } \\
\text { connectivity by } 8 \%\end{array}$ \\
\hline $\begin{array}{l}\text { Touati } \\
\text { et al. [14] }\end{array}$ & $\begin{array}{l}\text { CoMP for multipoint cooperative } \\
\text { amongst cell-edge users }\end{array}$ & $\begin{array}{l}\text { 5G system level in uplink and downlink } \\
\text { communication }\end{array}$ & $\begin{array}{l}\text { Enhance the throughput performance } \\
\text { in macromacro and macropicocells }\end{array}$ \\
\hline $\begin{array}{l}\text { Chu et al. } \\
{[15]}\end{array}$ & Joint CoMP in dense IoT & Downlink model in hyper IoT dense traffic & Enhancement in traffic pattern by $18 \%$ \\
\hline $\begin{array}{l}\text { Zitoune } \\
\text { et al. [16] }\end{array}$ & JT-CoMP OFDMA & The distributed density of nodes in eNB network & $\begin{array}{l}\text { Tolerant the spectrum efficiency } \\
\text { computing for the IoT nodes }\end{array}$ \\
\hline $\begin{array}{l}\text { Ali et al. } \\
{[17]}\end{array}$ & $\begin{array}{l}\text { Optimum aggregation of IoT data } \\
\text { network }\end{array}$ & $\begin{array}{l}\text { For cluster-based IoT by utilizing the heuristic- } \\
\text { based ILP technique }\end{array}$ & $\begin{array}{l}\text { Up to } 40 \% \text { throughput enhancement } \\
\text { with acceptable ILP complexity }\end{array}$ \\
\hline $\begin{array}{l}\text { Dai et al. } \\
{[18]}\end{array}$ & $\begin{array}{l}\text { DL (JT-NOMA-CoMP) model in } \\
\text { multiple cell networks }\end{array}$ & $\begin{array}{l}\text { Distributed power allocation for CoMP } \\
\text { orthogonal multiaccess (CoMP-OMAs) system }\end{array}$ & $\begin{array}{l}\text { Enhance the spectrum efficiency by } \\
\qquad 35 \%\end{array}$ \\
\hline $\begin{array}{l}\text { Al-Eryani } \\
\text { et al.[19] }\end{array}$ & $\begin{array}{l}\text { NOMA-CoMP-based IoT nodes } \\
\text { for interference mitigation }\end{array}$ & IoT based on grouping APs in a cluster & $\begin{array}{l}\text { Improves the total transmit power } \\
\text { (TP) for AP clusters }\end{array}$ \\
\hline $\begin{array}{l}\text { Chen } \\
\text { et al.[20] }\end{array}$ & $\begin{array}{l}\text { Generalized JT-CoMP with } \\
\text { NOMA }\end{array}$ & $\begin{array}{c}\text { UE outage probability and outage capacity with } \\
\text { various cooperative eNBs }\end{array}$ & Enhance the spectral efficiency \\
\hline $\begin{array}{l}\text { Rehman } \\
\text { et al. [21] }\end{array}$ & $\begin{array}{l}\text { Selective transmissions (STs) and } \\
\text { COMP-NOMA }\end{array}$ & Cell-edge user equipment- (UEs-) based NOMA & $\begin{array}{l}\text { Outperforms of ST-NOMA scheme } \\
\text { and high system throughput }\end{array}$ \\
\hline
\end{tabular}

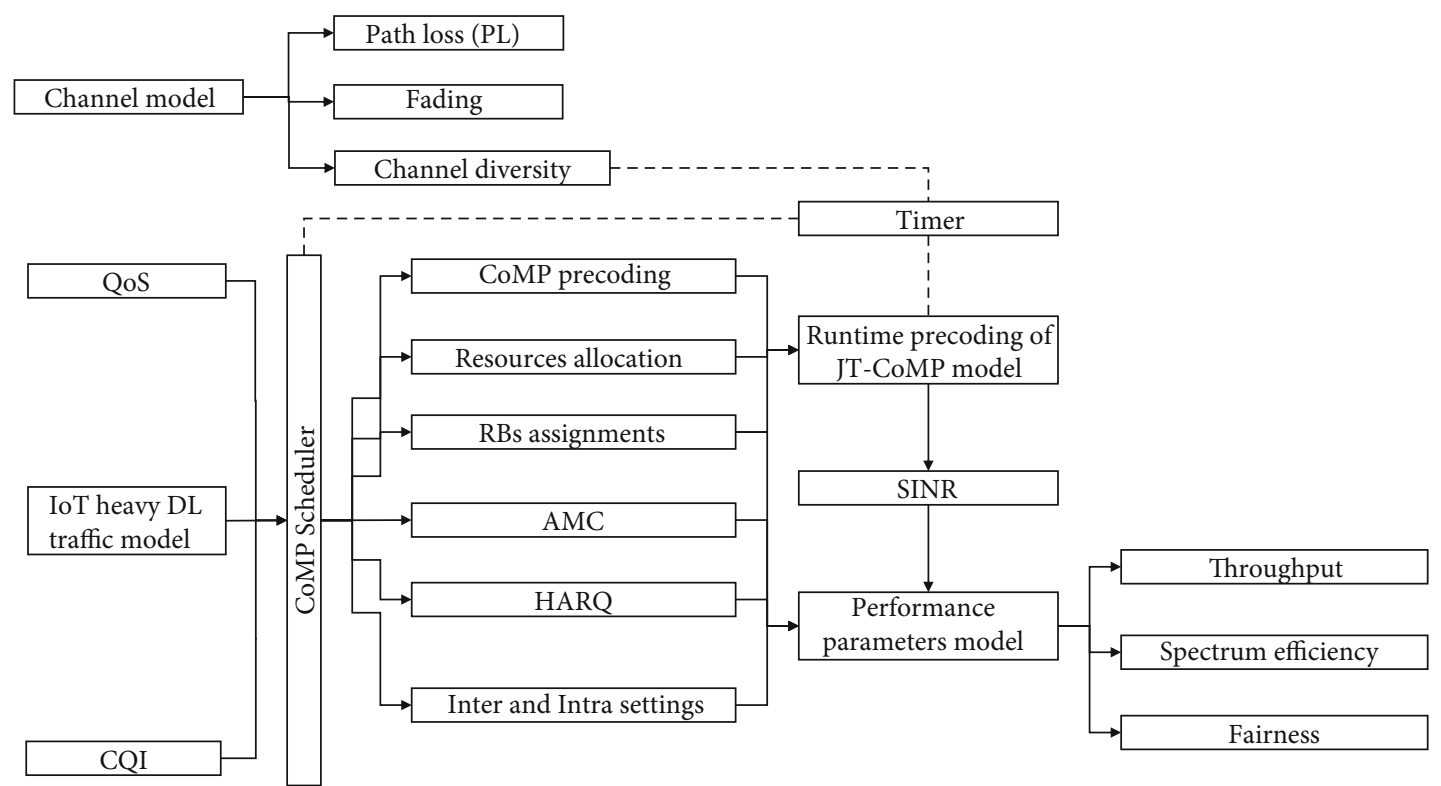

FIGURE 4: The abstraction structure of with proposed runtimes precoding of JT-CoMP model.

improvement in MIMO, and implementation of relay node (RN) [3].

CoMP is one of the most significant technical enhancements added to Release 18 that can be implemented in heterogonous networks (HetNets) implementation approaches and the homogenous networks' topologies. Nevertheless, with heterogonous and homogenous network deployment strategies and at most cell edge users are suffering from ICI [4], the purpose of the CoMP technique is to minimize ICI for cells that are using the same spectrum which is becoming more important and severe with the deployment of HetNet targeted by many network operators. CoMP shall enhance the transmit and receive to/from multipoints, which could be either multi- ple e-Node-Bs or remote radio heads (RRHs) [5]. CoMP enables transmission or reception to/from UEs, allowing the UE to select the closest eNB. The eNB is known as evolved node $\mathrm{B}$ or Evolved Universal Terrestrial Radio Access (EUTRAN) Node B, which would affect in consumption of power as well as increase the overall system performance and capacity. It furthermore balances the IoT heavy traffic load, exploits interference signal as a meaningful signal by using a JT mechanism or avoiding ICI by using a coordinated scheduling/beamforming (CS/CB) technique, and thus participates in mitigation of ICI [6]. In DL-CoMP joint JTs, multiple transmission points coordinate to transmit the same data to one UE in the same resources simultaneously. 


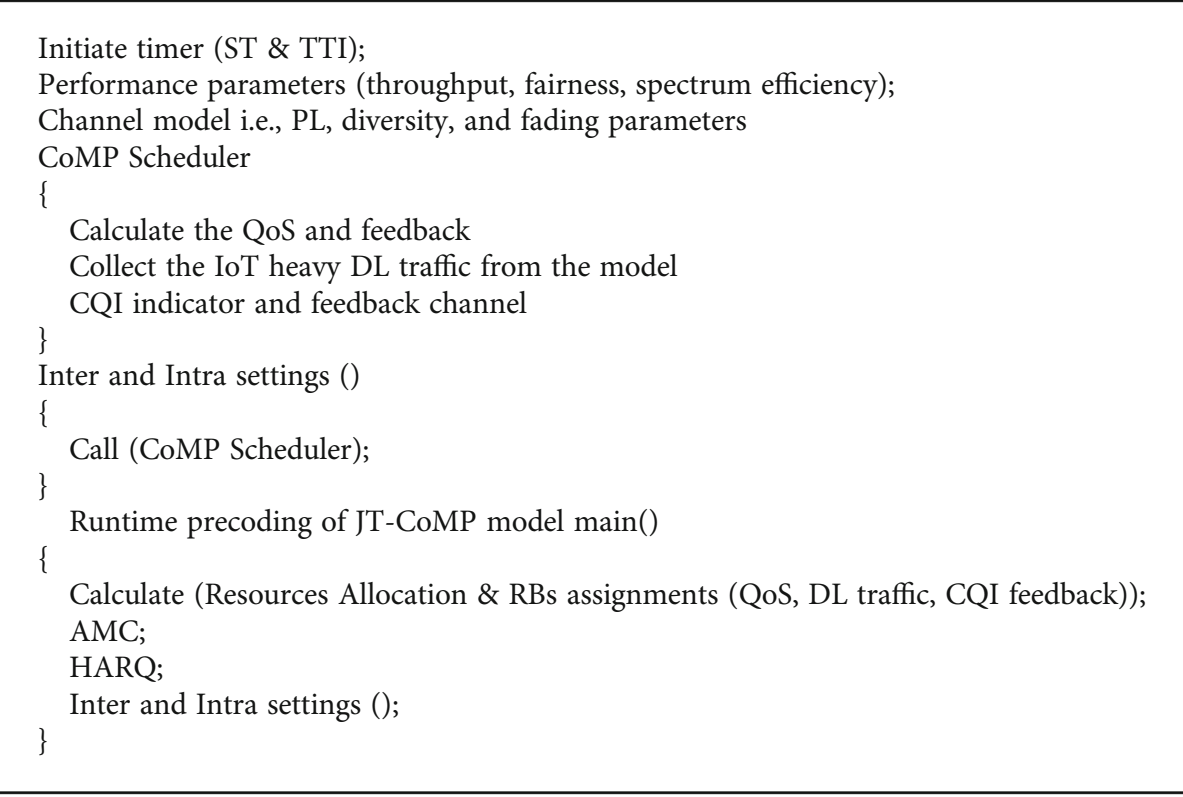

Algorithm 1: Proposed inter- and intrasettings with runtime precoding of JT-CoMP model.

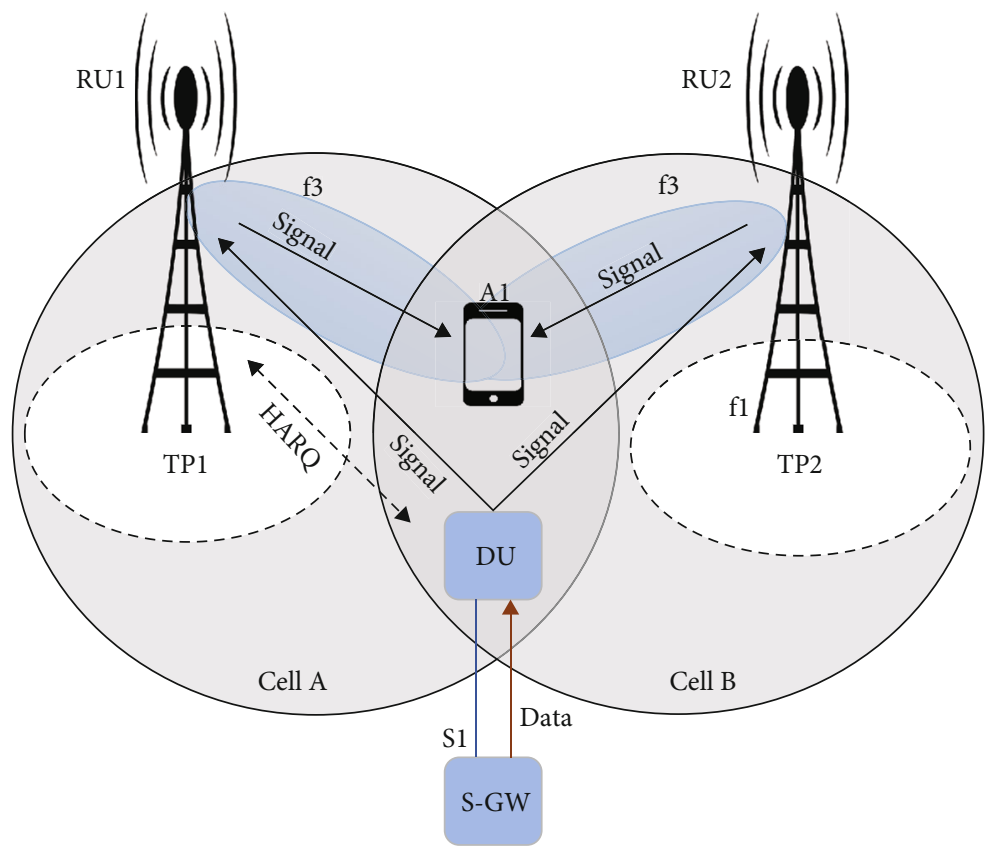

Figure 5: Intra-eNB joint transmission model and settings.

The main motivation for addressing the massive 5G-IoT devices scenario with heavy traffic load is that most cell edge IoT users are severely suffering from intercell interference (ICI), where the users have poor signal, lower data rates, and limited QoS [7]. This work purposes to address this problematic issue by proposing two types of DL-JT-CoMP techniques in 5G-IoT that are compliant with release 18 [8]. Downlink JT-CoMP with two homogeneous network CoMP deployment scenarios is considered and evaluated. The scenarios used are IoT intrasite and intersite CoMP which performance evaluated using downlink systems-level simulator for LTE-A and 5G. The paper contribution was shown in the simulation scenarios under high dense scenarios-with IoT heavy traffic load [9]. It has been shown that intersite CoMP has a better fairness index than intrasite CoMP by $5 \%$. The fairness index decreases when the users' number increase since the competition among users is higher. The intersite CoMP has better ECDF of average UE throughput than intrasite. Also, CoMP intersite CoMP has better ECDF of average UE spectral efficiency than intrasite CoMP, while the intersite CoMP has approximately the same ECDF of average SINR as intrasite CoMP [10].

The reminder sections of this paper are structured as follows: Section II presented the related literature for DL-JT- 


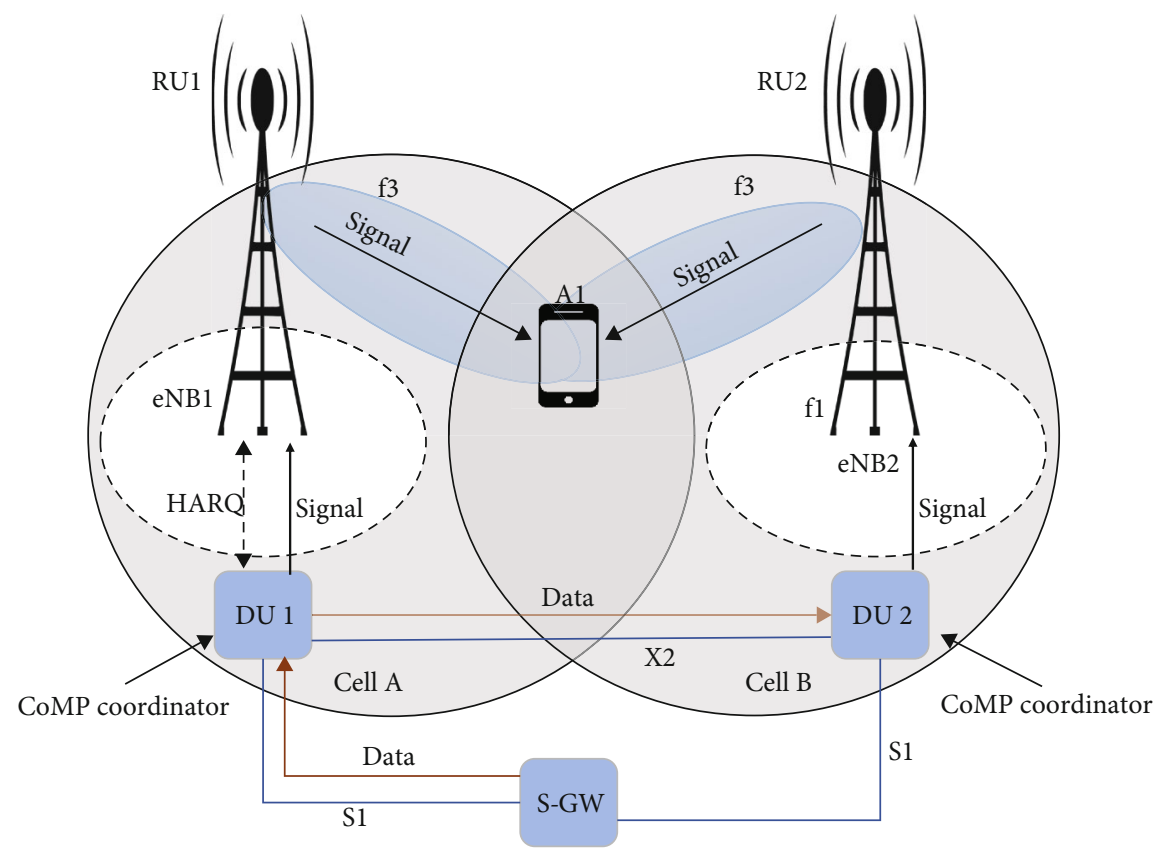

FIgURE 6: Inter-eNB joint transmission model and settings.

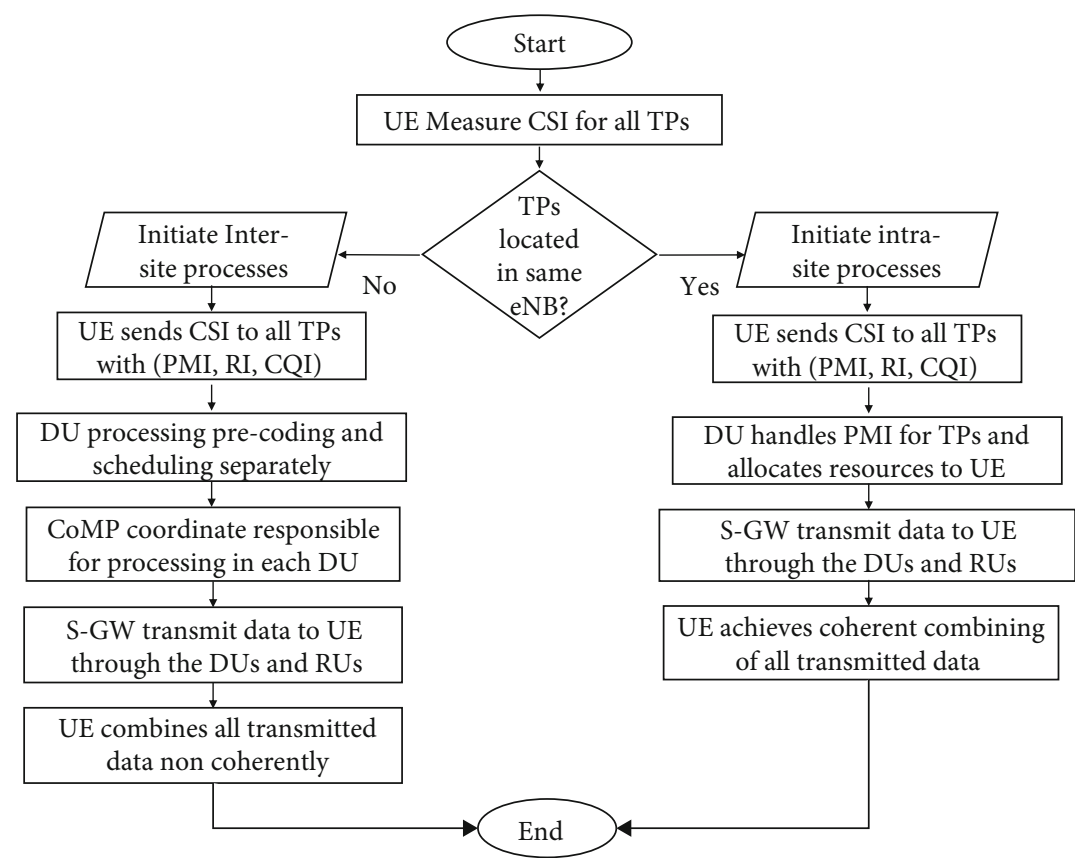

FIGURE 7: Algorithm for DL CoMP JT with intrasite and intersite IoT deployment scenarios.

CoMP, the proposed method, and the simulated scenarios that are discussed in Section III. Section IIV has illustrated the results from the system-level simulation. Finally, concluding remarks and future works have been given in Section $\mathrm{V}$.

\section{Background and Related Works}

The work of $5 \mathrm{G}$ is highly affected by the IoT intrasite and intersite interferences of neighboring devices. The interferences become severe in heterogeneous implementation as more IoT nodes are deployed especially in massive IoT scenarios, due to transmissions in cell boundaries. As a result, interference mitigation studies become crucial to ensure proper link quality for the data [11]. In this regard, the CoMP mechanism is working where received signals from neighbor eNBs are utilized to enhance network throughput. CoMP in 5G and LTE advanced has been extensively discussed in the literature, and Figure 1 shows the categorizations of CoMP based on DL and UL. The smallest timefrequency element that could be assigned to the UE in the 
$5 \mathrm{G}$ network is RBs. RBs are channels that involved OFDM set subcarriers for a certain period. 5G NRs allow for a huge block number of shapes extending from $480 \mathrm{kHz}$ down to $15 \mathrm{kHz}$ with a total physical layer of $5 \mathrm{MHz}$ [12], the 5Gdownlink frame, and radio resources that are illustrated in Figure 2. This research addressed the RB assignment to the UE problem for heavy IoT traffic load for downlink coordinated multipoint joint transmission. The $\mathrm{RB}$ is assigned at the start of all time slots based on each UE channel state.

$5 \mathrm{G}$ specification contains transmission time interval (TTI) scalability; according to slots duration $62.5 \mu \mathrm{sec}$ to $1 \mathrm{msec}$ (shown in Figure 3), 5G also includes minislot transmission, and this is same to short-TTI in LTE; sometimes, the simulation times is fixed to $\mathrm{n}$ TTI. In 5G, higher subcarrier spaces (SCSs) shorten the duration of symbols, which leads to shortened TTI. In contrast to LTE-A, 5G NRs enhance the TTI design to reduce latency and more radio resource efficiency. For ultrareliable and lower latency communications, (uRLLCs) low latencies are crucial in applications such as response to the disaster and automation of factories. Many types of research were investigated on the comprise CoMP in IoT, mobile, and cellular communications and evaluates its performance. Table 1 summarizes different related works and some recent researches on CoMP.

The authors in [13] proposed an analyzed technique for CoMP performance and deployment issues. The implemented scenarios show that the CoMP mechanism was useful in terms of throughput when applied to LTE-Advanced where the throughput was enhanced by $8 \%$ for downlink connectivity for each cell-edge user experience. Furthermore, the implemented scenario is considered realistic since the downlink traffic was in TTI burst format. CoMP performance evaluation was noticed for two realistic cell-edge users' scenarios for homogeneous and heterogeneous networks.

In [14], a demonstration of a CoMP throughput analysis for multipoint cooperative amongst cell-edge users and nodes is discussed. The $5 \mathrm{G}$ system-level simulations are implemented based HetNet scenario. A comparison study for various uplink and downlink scenarios was evaluated. The study was conducted in two networks i.e., macromacrocells and macropicocells. In both networks, the throughput performance was enhanced dramatically; especially in macropicocells where the edge users were highly overlapped between macrocell and picocell. In this paper, the CoMP is joint with precoding and scheduling techniques for both scenarios, which achieved additional throughput performance improvement.

The joint transmissions coordinated multipoint based on Release 15 specifications were implemented, analyzed, and evaluated in [15] using MATLAB ${ }^{\circledR}$. The cumulative distribution functions (CDF) and probability of outage with SNR and cell radius for adaptively shared downlink model to for hyper IoT dense traffic. The paper proposed a clustering technique for the IoT nodes based on their traffic pattern. The traffic pattern has been analyzed using a genetic algorithm. The achieved results were benchmarked with the performance of the conventional mechanism with no JT-CoMP, and apparent enhancements of $18 \%$ have been obtained.
TABLE 2: Simulation parameters.

\begin{tabular}{|c|c|}
\hline Parameters & Value \\
\hline Simulation type & tri_sector_tilted_4 $\times 4$ \\
\hline Sim-Set & {$\left[\begin{array}{lll}6 & 4 & 1\end{array}\right]$} \\
\hline $\mathrm{n}-\mathrm{RX}$ & Sim-Set (3) \\
\hline $\mathrm{n}-\mathrm{TX}$ & Sim-Set (2) \\
\hline Tx mode & Sim-Set (1) \\
\hline No. of transmit antenna port & 4 \\
\hline No. of received antenna port & 1 \\
\hline Number of eNodeB rings & 1 \\
\hline CoMP scheduler & RR-DB \\
\hline CoMP configuration & Intra, intersites \\
\hline eNodeB Tx power & $46 \mathrm{dBm}$ \\
\hline System frequency & $2.14 \mathrm{G}$ \\
\hline Bandwidth BW & $5 \mathrm{MHz}$ \\
\hline Channel delay & $3 \mu \mathrm{s}$ \\
\hline Simulation time (ST) & $4 \mathrm{~s}$ \\
\hline Transmission time interval (TTI) & $1 \mathrm{~ms}$ \\
\hline Intersite distance & $500 \mathrm{~m}$ \\
\hline IoT nodes per eNodeB & $10,30,60$ \\
\hline
\end{tabular}

The authors in [16] proposed a spatial modeling and mathematical framework using Monte Carlo simulations for complexity reduction for evaluating JT-CoMP OFDMA downlink. The paper considered a variety of spatially distributed density of nodes in a certain region of the eNBs network. It proposed a closed-form mathematical formula of three cases of interference based on coordinated eNB number. These obedient formulas tolerate hastily spectrum efficiency computing for the IoT nodes. One of the raised issues of the paper is that the model-based fluid did not catch the edge parts of the hexagon cell. Other issues like sharing data and backhaul synchronization are not assumed in the study.

In [17], optimum aggregation of IoT data network is proposed, where a cluster of IoT nodes is structured utilizing heuristic-based ILP (integer linear programming) technique. The proposed scheme obtained up to $40 \%$ throughput enhancement with acceptable ILP complexity. The grouping for the cluster in the proposed methods is assumed static; the work needs to be updated for dynamic and more realistic traffic patterns.

In [18], proposed optimum distributed power allocation for DL (JT-NOMA-CoMP) model in multiple cell networks; an optimal combined power allocation was articulated with closed form. Two-tier HetNet comprises the macrocell, which is underpaid, with some small cells with low-power users carrying similar RBs. The solutions are derived for all CoMP-sets containing multiple cooperative BSs (CoMPBS). The proposed method reveals substantial gains in energy and spectral efficiencies in disparity to legacy CoMP Orthogonal Multi-Access (CoMP-OMAs) system, where up to $25.5 \mathrm{bits} / \mathrm{sec} / \mathrm{Hz}$ spectrum efficiency is enhanced by $35 \%$ compared to the conventional JT-CoMP OMA. Energy 


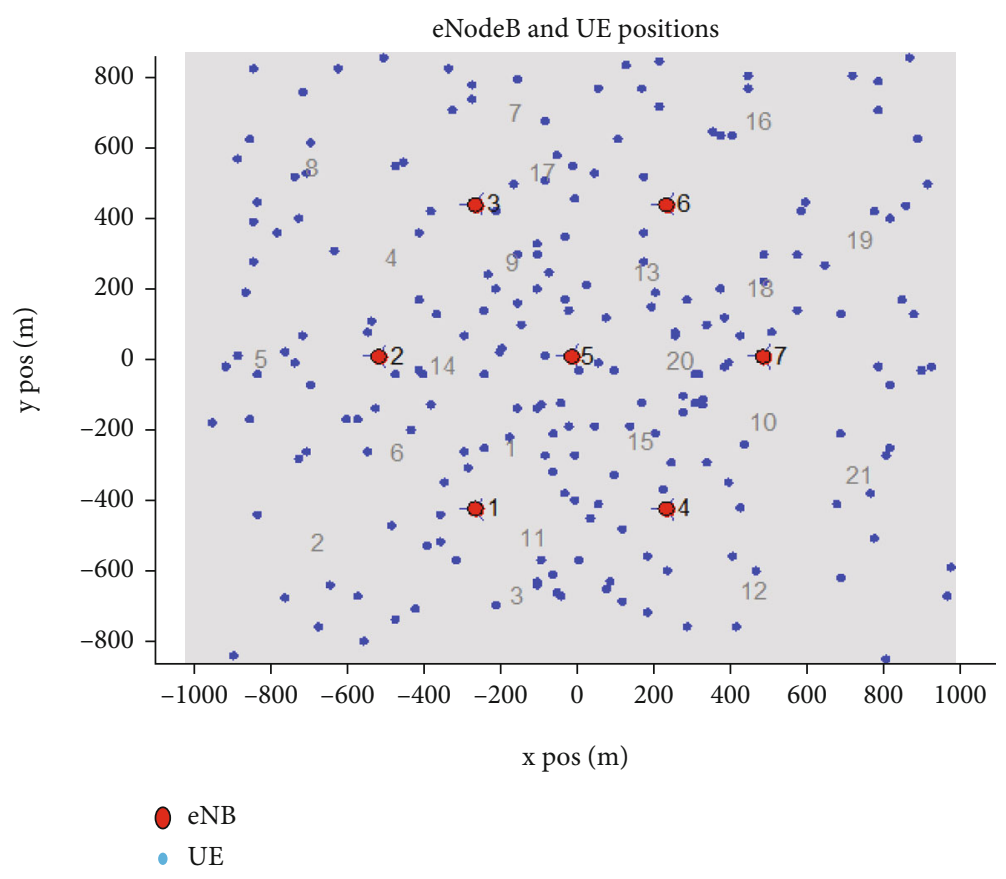

FIgURE 8: Random eNB and UE for IoT deployment for heavy load scenario.

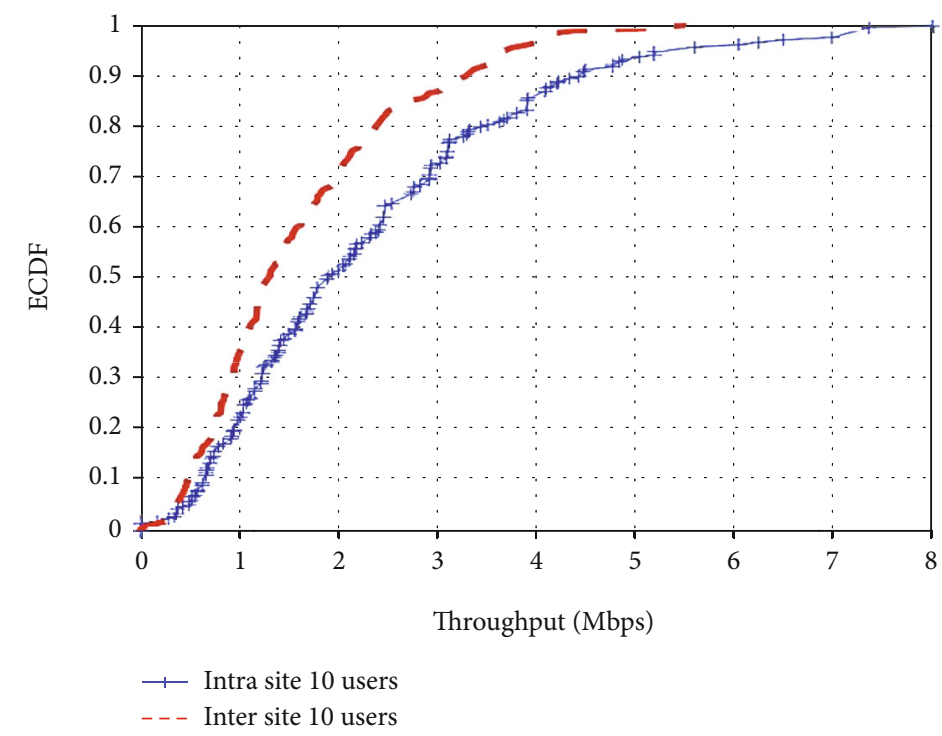

FIGURE 9: ECDF of UE throughput for intrasite and intersite CoMP with 10 IoT users/cell.

efficiency was $55 \mathrm{Mb} / \mathrm{J}$ with $28 \%$ enhancement compared to the conventional JT-CoMP OMA.

In [19], an exchange match-based model is employed for grouping APs in a cluster. AP cluster was proposed for optimum power control for NOMA-CoMP-based IoT nodes for interference mitigation. An iterative update for the AP clusters is applied. The proposed scheme achieved $3 \mathrm{~mW}$ total transmit power (TP) for 14 AP clusters while the conventional OMA was $15 \mathrm{~mW}$. For the $7 \mathrm{~dB}$ SINR threshold, the total TP was $20 \mathrm{~mW}$ for the proposed JACPC while the conventional OMA was $40 \mathrm{~mW}$. The computation overhead for the clusters iterative update did not discuss in the paper.
In [20], a new generalized JT-CoMP with NOMA scheme is proposed. To analyze the GCoMP-NOMA scheme, the paper proposed a closed-form formula for UE outage probability and outage capacity with various eNBs cooperative by considering both ideal and faded channelstate information (CSIs). The spectral efficiency for the proposed GCoMP-NOMA was $57 \mathrm{~b} / \mathrm{s} / \mathrm{Hz}$ while the conventional CoMP-OMA gave about $34 \mathrm{~b} / \mathrm{s} / \mathrm{Hz}$ (i.e., the BS power budget assumed to be $70 \mathrm{dBm}$ ).

In [21] that presented selective-transmissions (STs) for downlink COMP-NOMA networks, static and dynamic power allocation techniques were applied for various ST- 


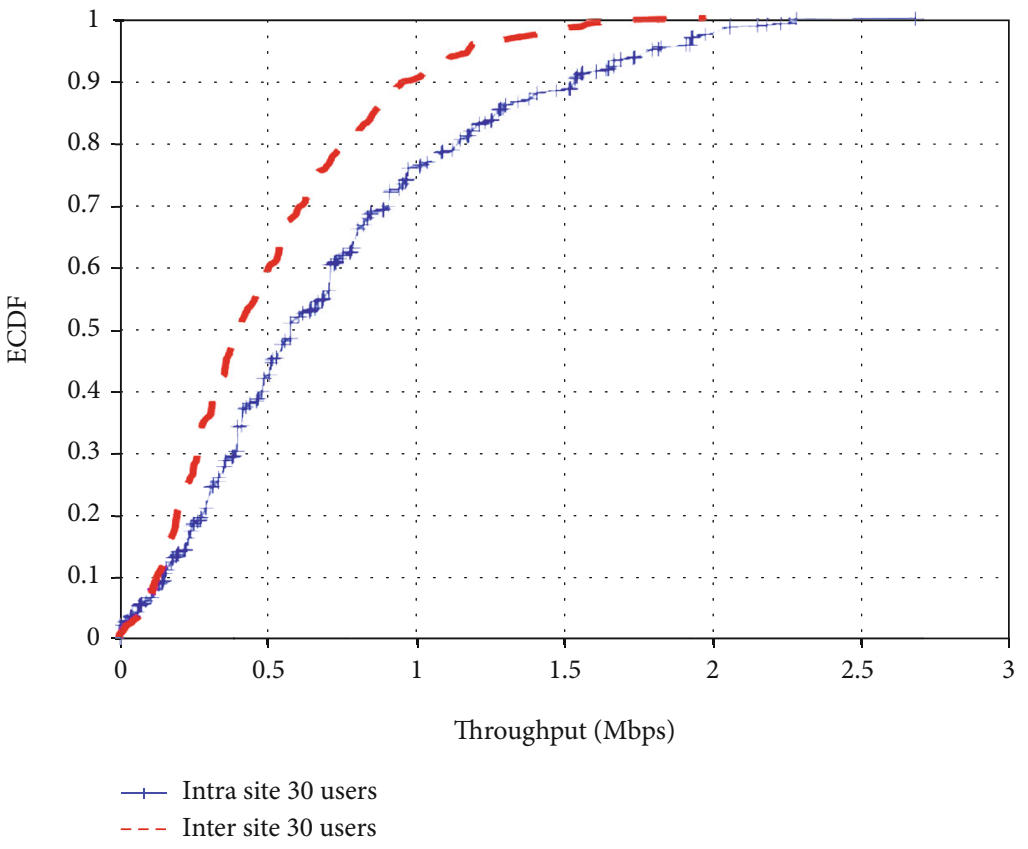

FIGURE 10: ECDF of UE throughput for IoT intrasite and intersite CoMP with 30 users/cell.

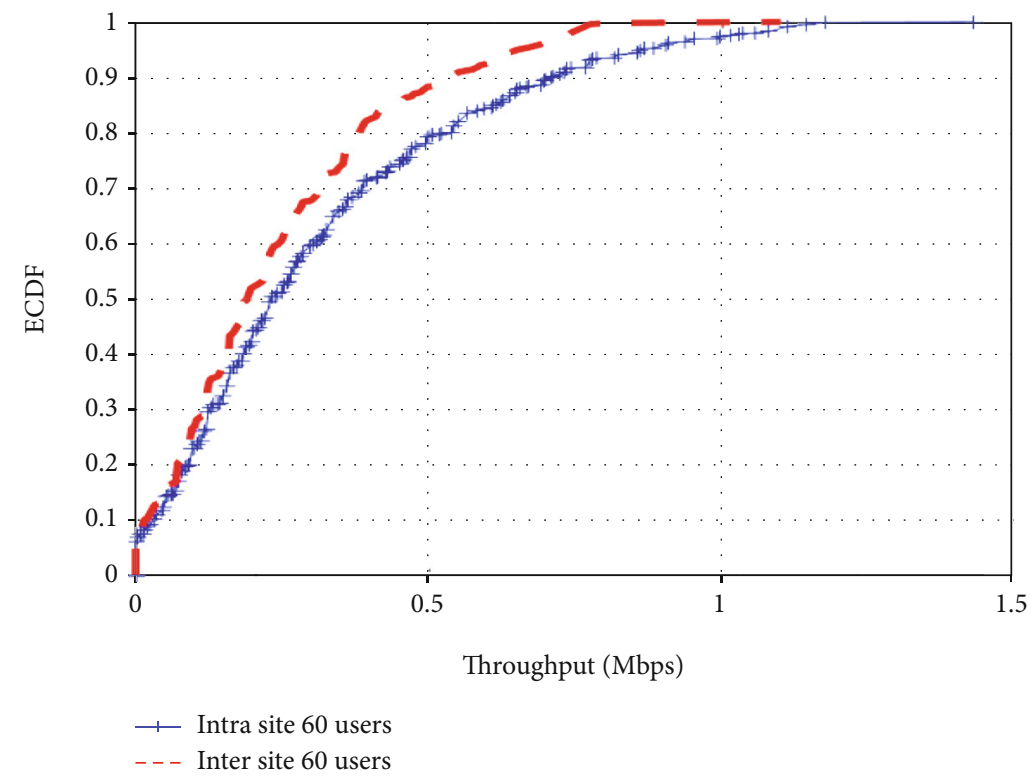

FIgURE 11: ECDF of UE throughput for IoT intrasite and intersite CoMP with 60 users/cell.

NOMA and conventional JT-NOMA. Optimum users' fairness is modeled as performance parameters where the fairness index was 0.78 for ST-NOMA and 0.86 for the conventional JT-NOMA. The spectral efficiency was $8 \mathrm{~b} / \mathrm{s} /$ $\mathrm{Hz}$ and $5.6 \mathrm{~b} / \mathrm{s} / \mathrm{Hz}$, respectively.

\section{The Methodology}

This paper is implemented 5G with DL-JT-CoMP technique then analyzes the performance using downlink system-level simulators (SLSs) for Rel-v2-15-Q3-2017 [22] with
MATLAB. Mechanisms to study machine type communication (MTC) IoT devices are specified in release 18 by 3GPP for $5 \mathrm{G}$ where the opportunity of half-duplex link enhanced the required peak data rate and the required channel operation bandwidth from 1.4 to $5 \mathrm{MHz}$; in the simulation, we used $5 \mathrm{MHz}$ heavy IoT traffic load scenario downlink. A contribution is done at the simulator level to suit our DL-JTCoMP, where an object program is developed for intra and intersite CoMP at physical level simulation, and the structure is shown in Figure 4 and Algorithm 1. In this paper, our focus is on two scenarios of CoMP in homogeneous 


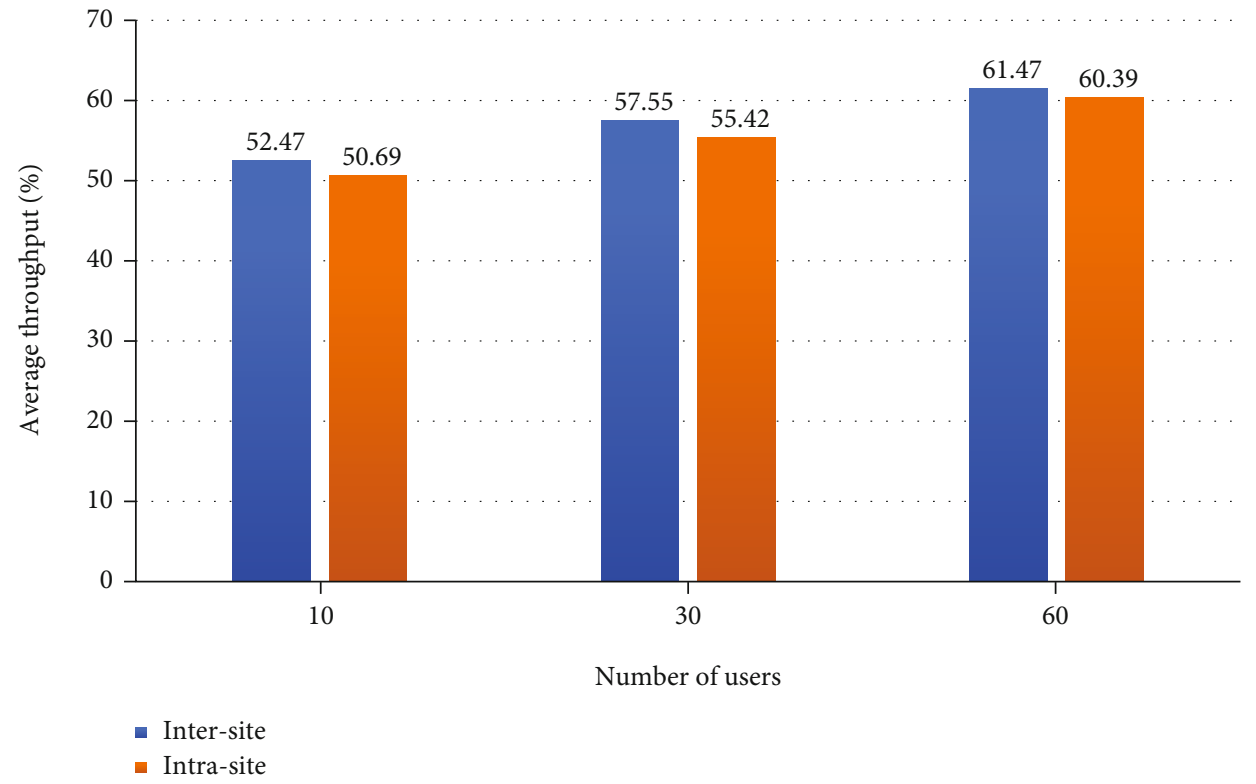

(a)

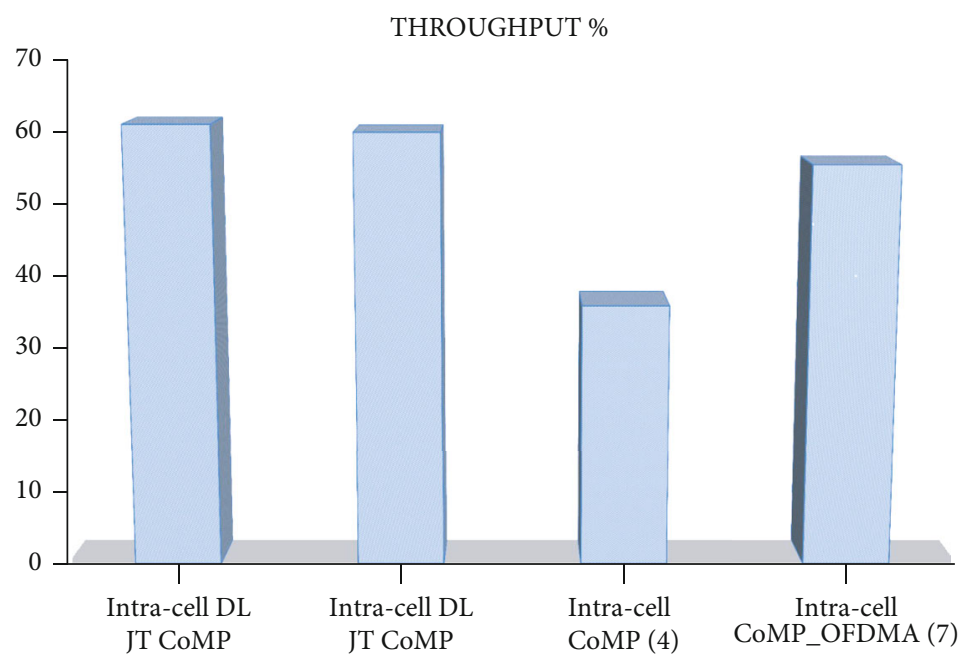

(b)

FIGURE 12: The average throughput percentage (a) proposed IoT intra and intersite scenarios (b) and compared with the proposed scheme with the related works in $[13,16]$.

deployment (intrasite and intersite) and then evaluates their performance in terms of UE throughput, UE spectral efficiency, SINR, and fairness index.

If the site has three sectors, then the number of coordinating cells in intrasite CoMP is also three [23]. The upgraded Vienna $5 \mathrm{G}$ model is employed for reciprocated databased exponent SINR-to-throughput mapping (RDESTM), which is compared with another conventional mapping in both performance and fairness. This scheme combines the information from IoT heavy DL traffic model, CQI, QoS, and channel model to the CoMP scheduler and JT-CoMP runtime model for both inter- and intrasite scenarios synchronized by a timer for transmission time intervals (TTI) and simulation time in the hardware environment [24].

In intrasite DL-JT-CoMP, the transmission points are belonging to the same eNB, and this eNB is controlled by a single digital unit (DU) also called baseband unit (BBU) as shown in Figure 5. Usually, it uses coherent transmission; in CJT, the UE can attain coherent combining of the transmission signals, and all TPs precode transmission matrix altogether. UE sends CSI (which contains CQI, RI, and PMI) to all cooperating TPs. All TPs alternately handle PMI to allocate the resources to UE and send it to that UE [25].

In intrasite CoMP, the sectors of the same eNB represent coordinating TPs. In intrasite, there is no need for an interface between TPs because they belong to the same eNB. In intersite DL-JT-CoMP, the transmission points are belonging to the different eNBs, and these eNBs are controlled by various DUs as shown in Figure 6. UE sends CSI (which contains CQI and RI) to all cooperating eNBs. When the scheduling and resource block (RB) allocation process fulfill 


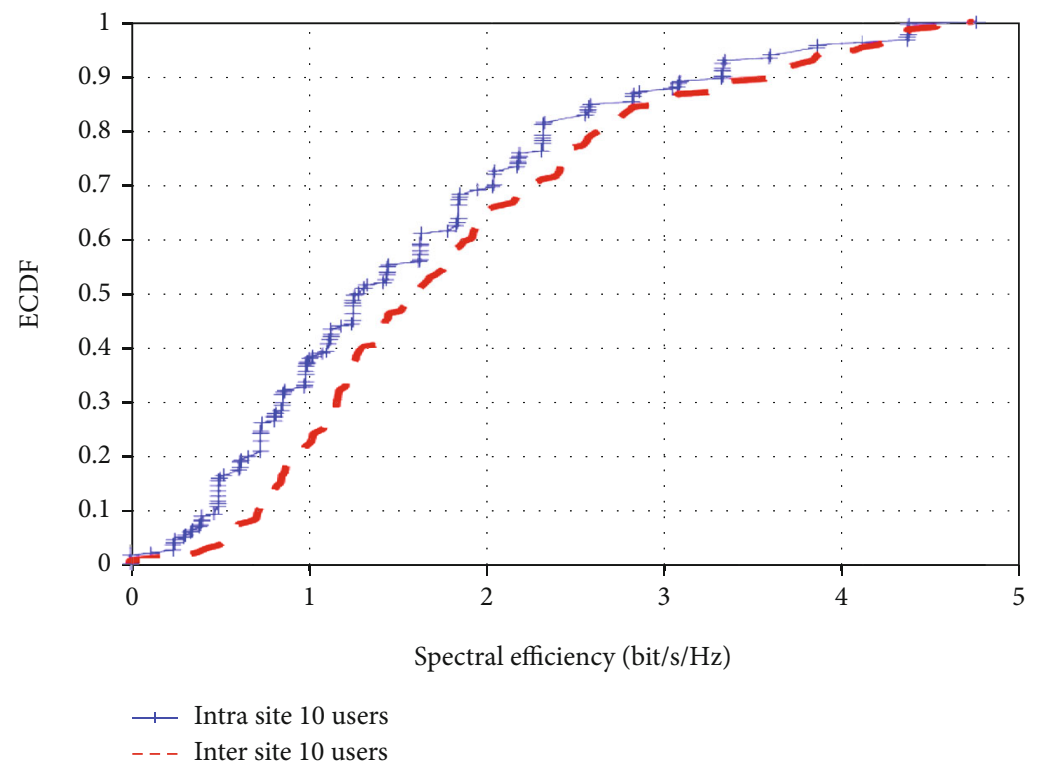

FIGURE 13: ECDF of UE spectral efficiency for intrasite and intersite with 10 IoT users/cell.

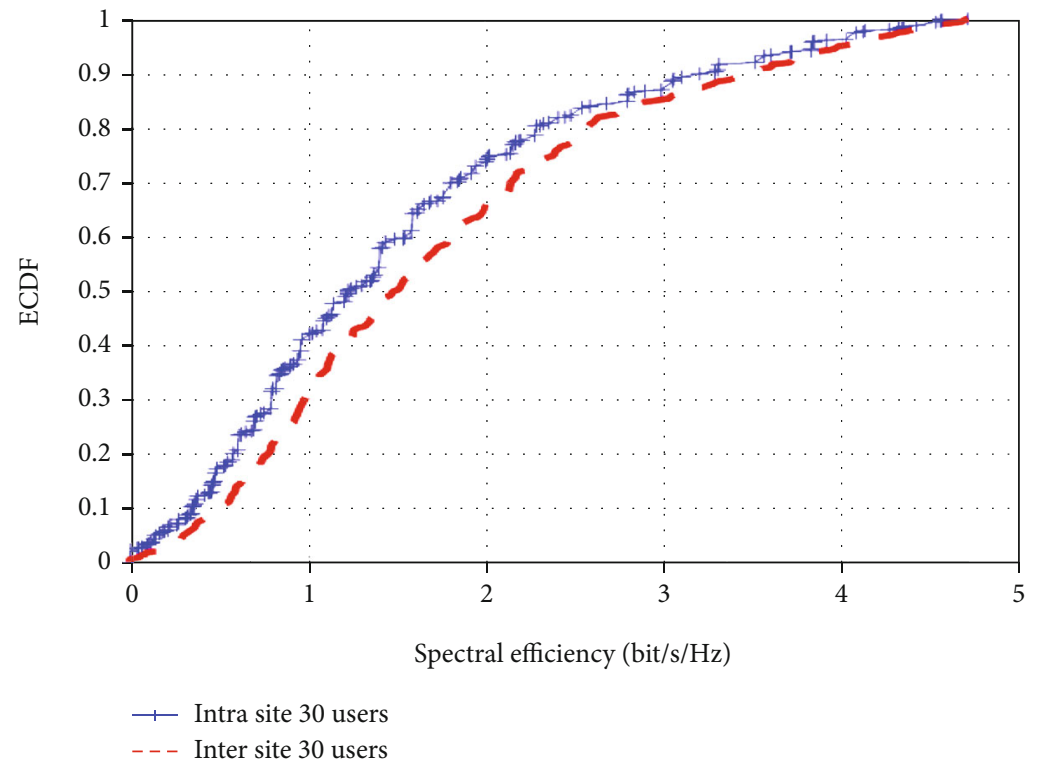

FIGURE 14: ECDF of UE spectral efficiency for intrasite and intersite with 30 IoT users/cell.

independently at the TP, distributed coordination is implemented in the network. Intersite coordination usually uses noncoherent transmission; In NCJT, each TP precodes the transmission matrix separately [26].

In intersite CoMP, the TPs represent the sectors of multiple eNBs. Then, the UE receives signals from multiple TPs. In the simulation, the TPs with the highest received signal power (that means higher SINR and then better CQI) are considered as the cooperating set. Figure 7 shows the flow chart for DL CoMP JT with IoT intrasite and intersite deployment scenarios.

In $5 \mathrm{G}$, the SINR is evaluated by UE on each PRB, then transforms to CQI, and reports it to eNB. CQI is used to select the most convenient modulation and coding schemes (MCS) for user data transmissions in a specific PRB. SNR is a good indicator for signal quality and is one of the most important factors that affect spectral efficiency and throughput [27]. The SINR for user $k$ in the system using a conventional $5 \mathrm{G}$ transmission is

$$
\mathrm{SINR}=\frac{P_{\mathrm{ue}} P L_{k, \mathrm{l}}}{P_{\mathrm{ue}} \sum_{i=1, i \neq 1}^{L} \mathrm{PL}_{i}+N},
$$

where $P_{\mathrm{ue}}$ is the transmitted power, $P_{\mathrm{Lk}, \mathrm{l}}$ is the path loss for user's $k$, and $N$ is the noise power. Spectral efficiency is 


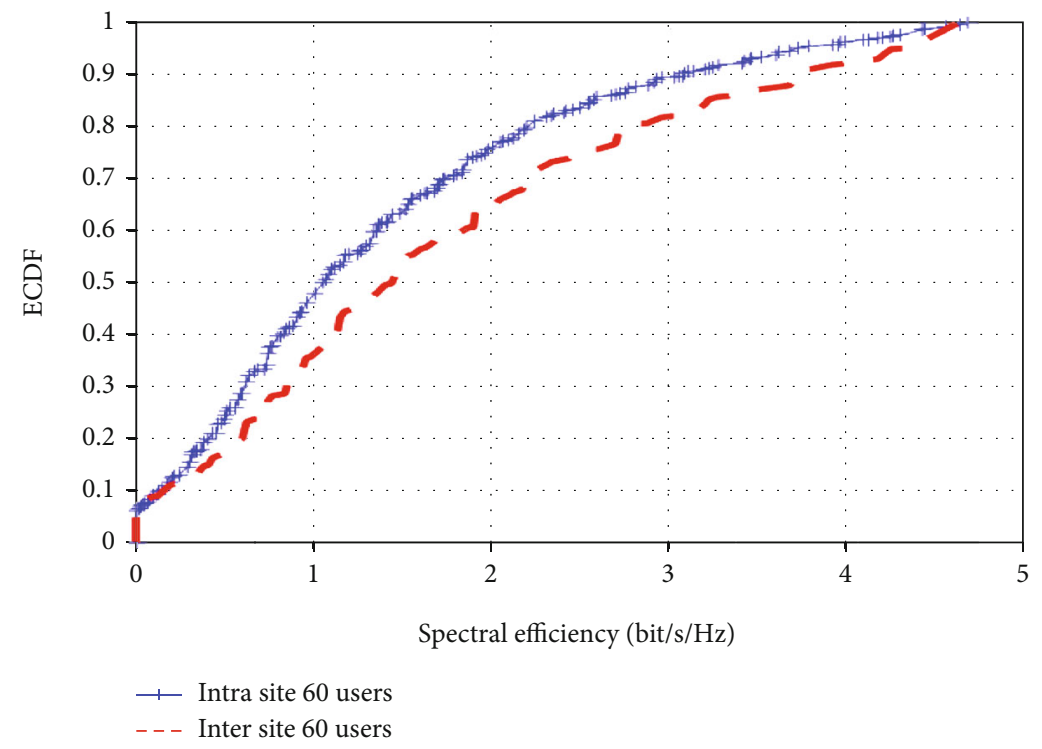

FIGURE 15: ECDF of UE spectral efficiency for intrasite and intersite with 60 IoT users/cell.

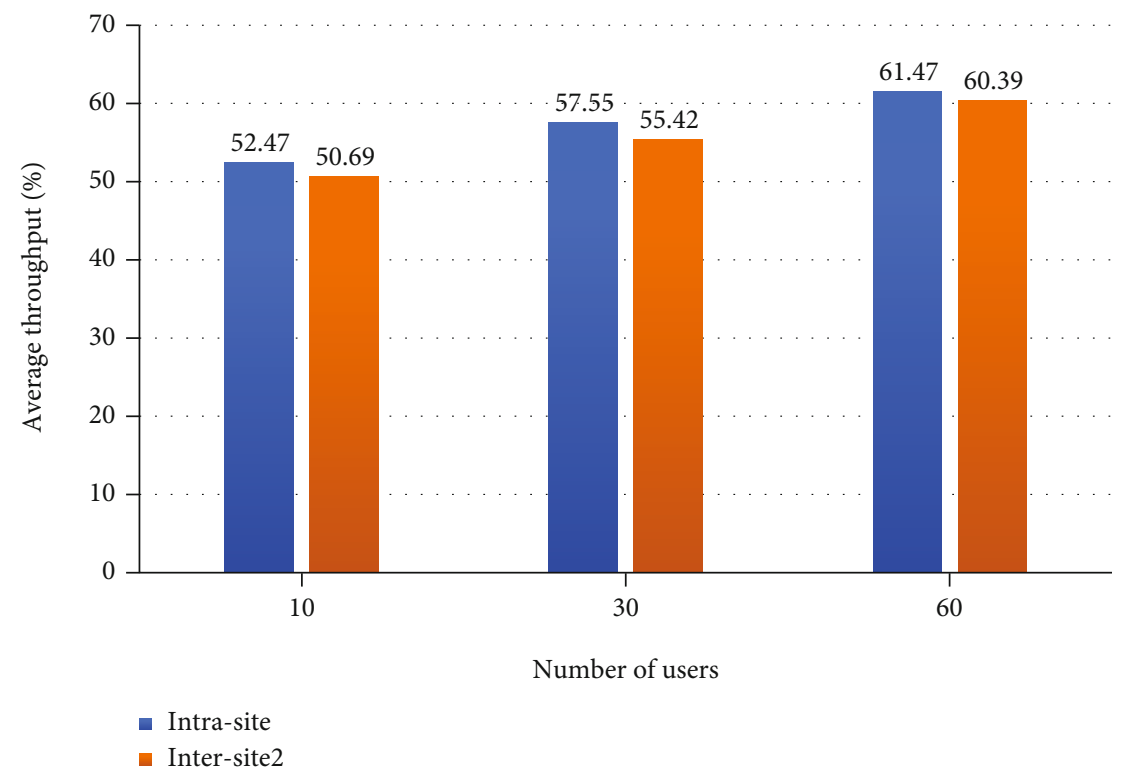

FIGURE 16: The average spectral efficiency percentage for IoT intra- and intersite scenarios.

the cell performance divided by the bandwidth [28]. The peak spectrum efficiencies are the maximum theoretical data rates divided by bandwidth when all PRBs are allocated to a single UE. The maximum spectral efficiency is achieved when the PRBs assign to the appropriate user, which has data to transmit within the cell [29]. The improved Shannon capacity expression aims to calculate the bandwidth efficiency of the system and the 5G SNR efficiency. The spectral efficiency utilizing the improved Shannon expression for user's $k$ can be written as $[14,30]$

$$
C(k)(\text { in bits } / \mathrm{s} / \mathrm{Hz})=\mathrm{BW}_{\text {eff }} \log _{2}\left(1+\mathrm{SINR}_{k} / \mathrm{SINR}_{\text {eff }}\right),
$$

where $\mathrm{BW}_{\mathrm{eff}}$ and $\mathrm{SINR}_{\mathrm{eff}}$ are the bandwidth efficiency and the SINR efficiency implementation of 5G, respectively. The UE throughput is defined as the amount of data sent successfully to a UE in the downlink divided by the simulation time [31]. It is calculated as follows:

$$
\text { Throughput }_{k}(\text { bits/s })=\mathrm{BW}_{k} C(k) \text {, }
$$

where $C(k)$ is the spectral efficiency in $\mathrm{bps} / \mathrm{Hz}$, and $\mathrm{BW}_{k}$ is the bandwidth. Fairness index represented by the Jain's Fairness Index (FI) is written as

$$
\text { Fairness Index }(\mathrm{FI})=\frac{\left(\sum_{k=1}^{K} R_{k}\right)^{2}}{U \sum_{k=1}^{K} R_{k}{ }^{2}},
$$




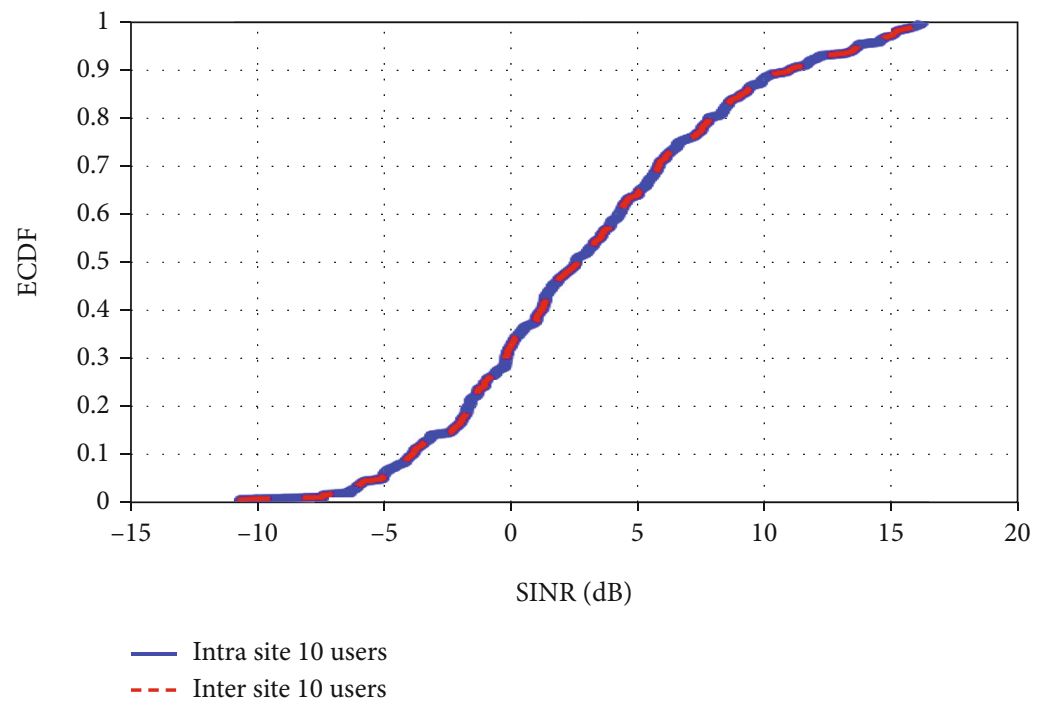

FIGURE 17: ECDF of UE SINR for intrasite and intersite with 10 IoT users/cell.

where $U$ is the number of IoT nodes in the network, and $R_{k}$ denotes the individual UE average throughput. FI indicates of fairness degree in the assigned rates or throughput performance between users in the network. The FI value ranges from zero to one, where $\mathrm{FI}=1$ means all IoT nodes have equal throughput on average [32]. In JT-CoMP, a UE is served by multiple TPs depending on the received signal powers from the different transmitters. The received signal at the UE using JT-CoMP is written as follows.

$$
\mathrm{P}_{r(k)}=\mathrm{P}_{\mathrm{ue}} \sum_{l \in L^{\prime}} \mathrm{PL}_{k, l}+\mathrm{P}_{\text {ue }} \sum_{j \in I \backslash L^{\prime}} \mathrm{PL}_{k, j}+N,
$$

where the first summation in the above equation represents the useful received signal from the $L^{\prime}$ cooperating transmits power (TPs), the second summation represents the interference signals, and $N$ is the noise power. Then, the SINR for the UE using JT-CoMP can be written as [33]

$$
\operatorname{SINR}_{\mathrm{k}}^{\text {CoMP JT }}=\frac{\sum_{\mathrm{l} \in \mathrm{L}^{\prime}} \mathrm{P}_{\mathrm{kl}}}{\sum_{\mathrm{j} \in \mathrm{l} \backslash \mathrm{L}^{\prime}} \mathrm{P}_{\mathrm{kj}}+\mathrm{N}},
$$

where $L^{\prime}$ is the subset of $L$ and consists of TPs for a UE. Empirical cumulative distribution function (ECDF) is a step function with $1 / n$ amplitude per $n$ data points, as

$$
[f, x]=\operatorname{ecdf}(y)
$$

where $f$ is evaluated at the points in $x$, using the data in the vector $y$. In the simulation, the ECDF is simulated to analyze the average UE throughput and spectral efficiency [34]. The computational complexity for inter- and intrasite process shows in Eq. (7) which it is acceptable for $f$ and $x$ as input and $y$ as a single output. Time complexity is considered high for the Algorithm 1, where Call (CoMP Scheduler); it may need to be iterated in nested manner for joint transmission especially for the UEs with high mobility sce- narios [17]. In the simulation, we noticed that some of the UEs with high mobility may not be capable for softhandover while inter/intrasite JT-CoMP algorithm is running.

\section{Results and Discussion}

In the performance evaluation, a JT-CoMP is enabled at system-level simulation. The UE downlink model of the Vienna 5G simulation [35] is extended by the inter- and intrasite functionalities supported by a timer for synchronization that is implemented in Algorithm 1. The simulator runtime with the conventional and contributed models was observed, and results were achieved.

For expressive benchmarking, the simulation environments were run in the same platform, i.e., an Intel Core-i7$10700 \mathrm{~K}, \mathrm{CPU}(3.80 \mathrm{GHz} 64 \mathrm{~GB}$ of DDR3 1600 integrated channels). The performance of CoMP transmission gain is presented in this section. The works conduct physical and system levels simulations with 3GPP radio access network (RAN) in [36]. It is also noted that the simulation result is evaluated by using a specific set of assumptions and parameters shown in Table 2. In addition, the evaluation is performed in both inter- and intrasite networks that are deployed within the macro-BS coverage area [37].

As shown in Figure 8, the graphical user interface (GUI) provides an idea about the location of the eNBs and the IoT nodes' positions. The numbers from 1 to 21 indicate to cells (TPs), and blue dots represent IoT nodes; red circles represent the seven sites eNBs. To evaluate the performance of DL-JT-CoMP, the simulation results of IoT intrasite and intersite will be compared in different performance metrics for a different number of users and show the effect of a different number of users in the performance metrics [38]. The results of performance metrics, throughput, spectral efficiency, and the wideband SINR were obtained based on the calculations during the use of three values of users' 


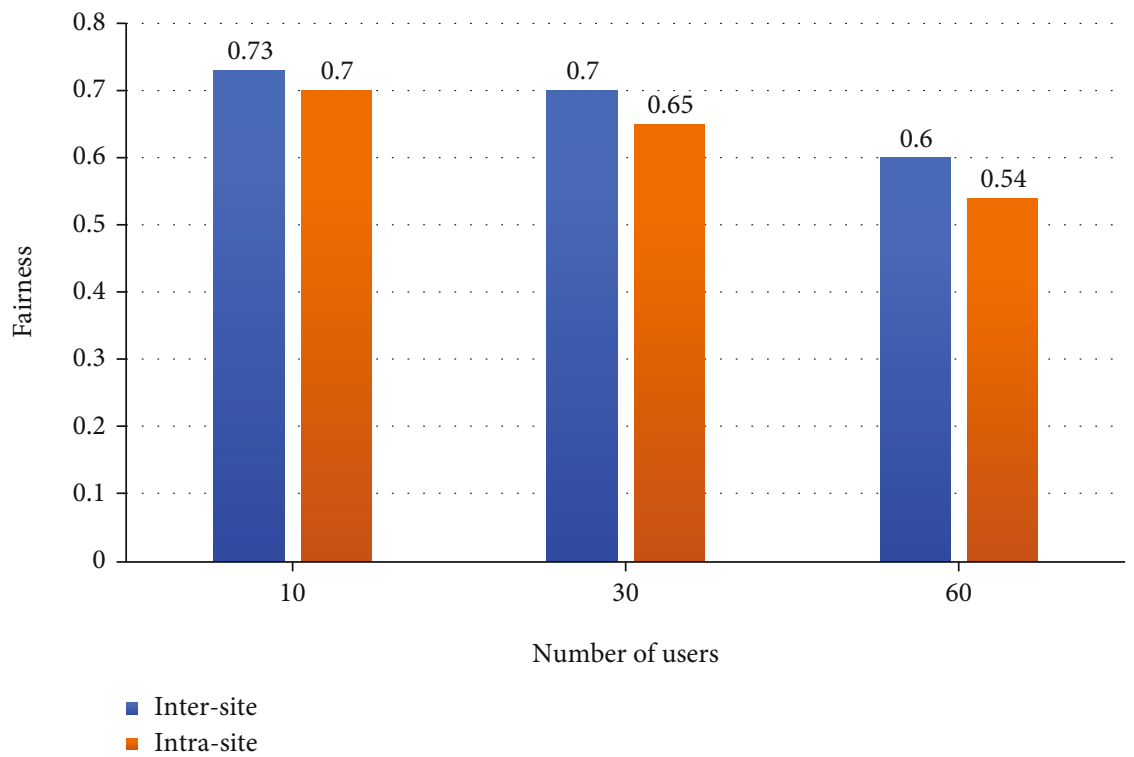

(a)

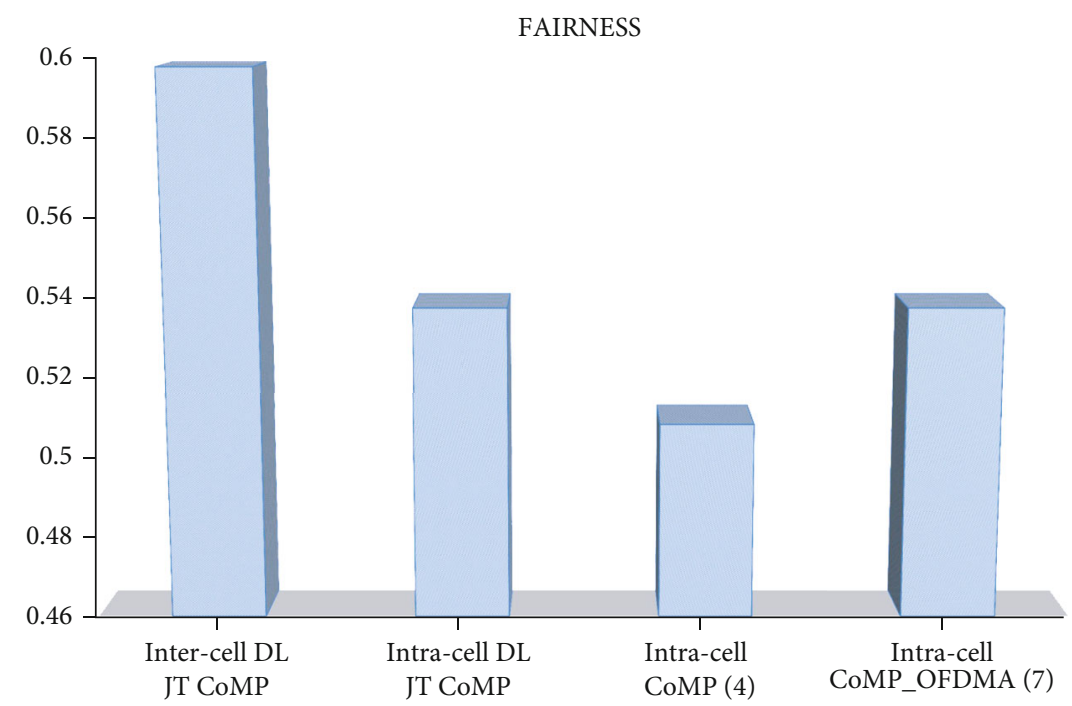

(b)

FIGURE 18: Fairness index in (a) the proposed scheme for intersite and intrasite CoMP with 10, 30, and 60 IoT users/cell (b) and compared the proposed scheme with the related works in $[13,16]$.

numbers, 10, 30, and $60 \mathrm{UE} /$ cell, respectively, to illustrate the ECDF for IoT intrasite and intersite UE throughput.

4.1. UE Throughput. As shown in Figures 9-11, the IoT intra- and intersites are evaluated based on the number of users in cells. The results show that an increase in the number of users within the cell will negatively affect the UE throughput for both cases because the network resources will be divided into a larger number when increasing the number of IoT nodes/cells. That means the higher number of users, the lower the throughput in the two cases of IoT intersite and intrasite CoMP.

As shown in Figure 12(a), the average throughput for the inter- and intrasties is increased according to the number of users. However, the comparison between the inter- and intrasites in terms of average percentages against the num- ber of users shows that when used 60 users/cell, the intrasite gives more ECPF than intersite and is dramatically affect the UE throughput. Figure 12(b) shows the average throughput percentage compared with the proposed scheme with the related works in $[13,16]$.

4.2. UE Spectral Efficiency. The evaluation related to the inter- and intrasites in terms of UE spectral efficiency is shown in Figures 13-15. In Figure 13, we observed that in the case of 10 users used in intersite, $50 \%$ of users have spectral efficiency less than or equal to $1.6 \mathrm{~b} / \mathrm{s} / \mathrm{Hz}$; however, in intrasite, $50 \%$ of users have spectral efficiency less than or equal to $1.2 \mathrm{~b} / \mathrm{s} / \mathrm{Hz}$.

In the case of 30 users used, intersites give that $50 \%$ of users have spectral efficiency less than or equal to $1.5 \mathrm{~b} / \mathrm{s} / \mathrm{Hz}$, and in intrasite, $50 \%$ of users have spectral efficiency less than 
TABLE 3: The UE wideband SINR percentage for IoT intra- and intersite scenarios.

\begin{tabular}{lcc}
\hline Number of IoT nodes & Intrasite & Intersite \\
\hline 10 & $50.02 \%$ & $50.02 \%$ \\
30 & $49.82 \%$ & $49.73 \%$ \\
60 & $50.06 \%$ & $49.93 \%$ \\
\hline
\end{tabular}

or equal to $1.1 \mathrm{~b} / \mathrm{s} / \mathrm{Hz}$. In the case of 60 users, the intersite gives $50 \%$ of users have spectral efficiency less than or equal to $1.4 \mathrm{~b} /$ $\mathrm{s} / \mathrm{Hz}$. However, in intrasite, $50 \%$ of users have spectral efficiency less than or equal to $1 \mathrm{~b} / \mathrm{s} / \mathrm{Hz}$ as illustrated in Figure 15 .

In Figure 16, we noticed that the increased number of users/cells would increase slightly the user's average spectral efficiency in both, intra- and inter sites. Accordingly, the percentage of users that have average spectral efficiency for intrasite is less than the percentage of users that have average spectral efficiency for intersite. In addition, when the number of users increased, the UE spectral efficiency decreased in the two cases of intersite and intrasite CoMP because the network resources will be divided on a larger number of users as in throughput [32].

4.3. UE Wideband SINR. The simulation results of UE wideband SINR in Figure 17 show that the user numbers of 10, 30 , and 60 users/cell give the same performance for both intersite and intrasites. The observed results show that both sites depicted $62 \%$ of users have SINR less than or equal to $5 \mathrm{~dB}$, and the SINR enhanced for $38 \%$ of the IoT nodes. The better SINR achieved is nearly up to $17 \mathrm{~dB}$. That means the number of users has little effect on the average of SINR in the two cases of intersite and intrasite CoMP as shown in Table 2.

4.4. Fairness Index. The fairness index for the different numbers of users per cell in intersite and intrasite CoMP is illustrated in Figure 18(a). It shows that the fairness index of intersite with 10,30, and 60 users/cell is better than in intrasite. Figure 18(b) shows the average throughput percentage compared the proposed scheme with the related works in $[13,16]$ (see Table 3 ).

We observed that, higher fairness index in intersite means that, the system attempts to maintain the UE throughput similar for all the IoT nodes in the network regardless of their locations relative to eNB and received SINR [33-35]. According to the fairness index for intraand intersites, a higher number of users gives less fairness index in the two cases of intersite and intrasite CoMP, because the competition between users becomes tenser.

\section{Conclusion}

This paper is mainly aimed at evaluating the performance of DL-JT-CoMP with two scenarios of CoMP homogeneous deployment. The scenarios used are IoT intrasite and intersite CoMP, which performance evaluated using downlink system-level simulator for long-term evolution-advanced (LTE-A) and 5G. The achieved results concluded that the intersite CoMP has better performance than the intrasite CoMP. The reason is that intersite CoMP has a better effect on more users where the cooperating cells are at different geographical locations (belong to different eNBs). In intrasite CoMP, the cooperating cells are in form of sectors for the same eNB, and the overlapping is quite limited. Future work and extension of this work are to study other COMP-based 5G techniques like MIMO with further enhanced (FE) for non-CA-based intercell interference coordination (ICIC) and evaluate their performance. In addition, one can compare joint transmissions (JTs) and coordinated scheduling/beamforming $(\mathrm{CS} / \mathrm{BF})$ in downlink and evaluate their performances.

\section{Data Availability}

The datasets generated during and/or analyzed during the current study are available from the corresponding author on reasonable request.

\section{Ethical Approval}

Hereby, the authors consciously assure that for the manuscript/insert title, the following is fulfilled: (a) this material is the authors' original work, which has not been previously published elsewhere. (b) The paper is not currently being considered for publication elsewhere. (c) The paper reflects the authors' research and analysis truthfully and completely. (d) The paper properly credits the meaningful contributions of coauthors and coresearchers. (e) The results are appropriately placed in the context of prior and existing research. (f) All sources used are properly disclosed (correct citation). Copying of text must be indicated as such by using quotation marks and giving proper reference. (g) All authors have been personally and actively involved in the substantial work leading to the paper and will take public responsibility for its content.

\section{Conflicts of Interest}

The authors declare that they have no known competing financial interests or personal relationships that could have appeared to influence the work reported in this paper.

\section{Acknowledgments}

This research was supported by Taif University Researchers Supporting Project number (TURSP-2020/216), Taif University, Taif, Saudi Arabia.

\section{References}

[1] A. Rajanna and M. Haenggi, "Downlink coordinated joint transmission for mutual information accumulation," IEEE Wireless Communications Letters, vol. 6, no. 2, pp. 198-201, 2017.

[2] H. Sun, W. Fang, J. Liu, and Y. Meng, "Performance evaluation of CS/CB for coordinated multipoint transmission in LTE-A downlink," in 2012 IEEE 23rd International Symposium on 
Personal, Indoor, and Mobile Radio Communications (PIMRC), pp. 1061-1065, Sydney, NSW, Australia, 2012.

[3] N. Nurelmadina, M. Hasan, I. Memon et al., "A systematic review on cgnitive radio in low power wide area network for industrial IoT applications," Sustainability, vol. 13, no. 1, p. 338, 2021.

[4] A. Hooshmand, H. Aghvami, and B. W. K. Ling, "Problem formulation for joint cooperative downlink scheduling and power allocation for joint processing coordinated multipoint," IEEE International Conference on Consumer Electronics - China, pp. 1-3, Shenzhen, China, 2014.

[5] M. B. Hassan, E. S. Ali, R. A. Mokhtar, R. A. Saeed, and B. S. Chaudhari, "6- NB-IoT: concepts, applications, and deployment challenges," in LPWAN Technologies for IoT and M2M Applications, B. S. Chaudhari and M. Zennaro, Eds., Academic Press, 2020.

[6] L. D. Palhão Mendes, L. Leonel Mendes, and T. C. Pimenta, "Uplink dynamic point blanking coordinated multipoint scheduler for IoT integration in $5 \mathrm{G}$ networks," IEEE $5 \mathrm{G}$ World Forum (5GWF), pp. 440-445, Silicon Valley, CA, USA, 2018.

[7] F. Alsolami, F. A. Alqurashi, M. Hasan, R. A. Saeed, S. AbdelKhalek, and A. Ben Ishak, "Development of self-synchronized drones' network using cluster-based swarm intelligence approach," IEEE Access, vol. 9, pp. 48010-48022, 2021.

[8] A. R. Oessler and M. Kottkamp, "LTE-advanced (3GPP Release 11) technology introduction, white paper," Rohde \& Schwarz, vol. 7, 2013.

[9] S. N. Ghorpade, M. Zennaro, B. S. Chaudhari, R. A. Saeed, H. Alhumyani, and S. Abdel-Khalek, "Enhanced differential crossover and quantum particle swarm optimization for IoT applications," IEEE Access, vol. 9, pp. 93831-93846, 2021.

[10] L. Zitoune, S. Cerovic, D. Cerovic, V. Vèque, and J. M. Kelif, "Performance evaluation of JT CoMP approach: tractable model using spatial fluid modeling," in 2016 IFIP Networking Conference (IFIP Networking) and Workshops, pp. 198-206, Vienna, Austria, 2016.

[11] M. Abdelgadir and R. A. Saeed, "Evaluation of performance enhancement of OFDM based on cross layer design (CLD) IEEE 802.11p standard for vehicular ad-hoc networks (VANETs), City Sce," International Journal of Signal Processing Systems, vol. 8, no. 1, pp. 1-7, 2020.

[12] A. M. Musa, R. A. Mokhtar, R. A. Saeed, H. Alhumyani, S. Abdel-Khalek, and A. Omer Yousif Mohamed, "Distributed SC-FDMA sub-carrier assignment for digital mobile satellite," Alexandria Engineering Journal, vol. 60, no. 6, pp. 4973-4980, 2021.

[13] X. Ouyang, C. Min Zhang, Z. Song, J. Wang, and Z. W. Li, "Service-aware dynamic bandwidth allocation with CoMP in 5G front haul network," 2017, pp. 1-3, Wuzhen, China, 2017 16th International Conference on Optical Communications and Networks (ICOCN),

[14] H. Touati, H. Castel-Taleb, B. Jouaber, and S. Akbarzadeh, "Model-Based optimization for JT CoMP in C-RAN," in IEEE INFOCOM 2019 - IEEE Conference on Computer Communications Workshops (INFOCOM WKSHPS), pp. 403-409, Paris, France, 2019.

[15] M. Chu, S. Moon, H. Choe, and I. Hwang, "Performance analysis of CoMP using scheduling and precoding techniques in the heterogeneous network," International Journal of Information and Electronics Engineering, vol. 6, no. 3, pp. 155-160, 2016.
[16] L. Zitoune, S. Cerovic, D. Cerovic, V. Vèque, and J. Kelif, "Performance evaluation of JT CoMP approach: tractable model using spatial fluid modeling," in 2016 IFIP Networking Conference (IFIP Networking) and Workshops, pp. 198-206, Vienna, Austria, 2016.

[17] E. S. Ali, M. K. Hasan, R. Hassan et al., "Machine learning technologies for secure vehicular communication in internet of vehicles: recent advances and applications," Security and Communication Networks, vol. 2021, 23 pages, 2021.

[18] M. S. Ali, E. Hossain, A. Al-Dweik, and D. I. Kim, "Downlink power allocation for CoMP-NOMA in multi-cell networks," IEEE Transactions on Communications, vol. 66, no. 9, pp. 3982-3998, 2018.

[19] Y. Dai and L. Lyu, "NOMA-enabled CoMP clustering and power control for green Internet of Things networks," IEEE Access, vol. 8, pp. 90109-90117, 2020.

[20] Y. al-Eryani, E. Hossain, and D. In Kim, "Generalized coordinated multipoint (GCoMP)-enabled NOMA: outage, capacity, and power allocation," IEEE Transactions on Communications, vol. 67, no. 11, pp. 7923-7936, 2019.

[21] J. Chen, X. Ge, Y. Zhong, and Y. Li, "A novel JT-CoMP scheme in 5G fractal small cell networks," in 2019 IEEE Wireless Communications and Networking Conference (WCNC), Marrakesh, Morocco, 2019.

[22] C. Liu and P. Chen, "Load-aware coordinated multipoint joint transmission in dense heterogeneous networks: downlink coverage and throughput limits," IEEE International Conference on Communications (ICC), pp. 1-7, Paris, France, 2017.

[23] B. U. Rehman, M. I. Babar, A. W. Ahmad et al., "Joint power control and user grouping for uplink power domain nonorthogonal multiple access," International Journal of Distributed Sensor Networks, vol. 17, no. 12, 2021.

[24] A. Chapnevis, İ. Güvenç, and E. Bulut, "Traffic shifting based resource optimization in aggregated IoT communication," in 2020 IEEE 45th Conference on Local Computer Networks (LCN), pp. 233-243, Sydney, NSW, Australia, 2020.

[25] R. A. Mokhtar, R. A. Saeed, H. Alhumyani, M. Khayyat, and S. Abdel-Khalek, "Cluster mechanism for sensing data report using robust collaborative distributed spectrum sensing," Cluster Computing, vol. 24, 2021.

[26] H. Li, "Joint dirty paper precoding and user scheduling for downlink coordinated multipoint transmission system," in 2009 5th International Conference on Wireless Communications, Networking and Mobile Computing, pp. 1-4, Beijing, China, 2009.

[27] A. A. Eltahir and R. A. Saeed, "V2V Communication Protocols in Cloud-Assisted Vehicular Networks," in Vehicular Cloud Computing for Traffic Management and Systems, IGI Global, 2018.

[28] H. Sun, W. Fang, and L. Yang, "A novel precoder design for coordinated multipoint downlink transmission," in 2011 IEEE 73rd Vehicular Technology Conference (VTC Spring), pp. 1-5, Budapest, Hungary, 2011.

[29] R. A. Saeed and E. B. Abbas, "Performance evaluation of MIMO FSO communication with gamma-gamma turbulence channel using dversity techniques," in 2018 International Conference on Computer, Control, Electrical, and Electronics Engineering (ICCCEEE), pp. 1-6, Khartoum, Sudan, 2018.

[30] M. F. Hamdi, R. A. Saeed, and A. S. Abas, "Downlink scheduling in 5G massive MIMO," Journal of Engineering and Applied Sciences, vol. 13, pp. 1376-1381, 2018. 
[31] R. K. Jain, D. W. Chiu, and W. R. Hawe, "A quantitative measure of fairness and discrimination for resource allocation in shared computer systems," Digital Equipment Corporation, Tech. Rep, 1984.

[32] Z. E. Ahmed, R. A. Saeed, and A. Mukherjee, "Challenges and opportunities in vehicular cloud computing," in Cloud Security - Concepts, Methodologies, Tools, and Applications, pp. 21682185, IGI Global, 2019.

[33] Z. E. Ahmed, R. A. Saeed, A. Mukherjee, and S. N. Ghorpade, "10- Energy optimization in low-power wide-area networks by using heuristic techniques," in LPWAN Technologies for IoT and M2M Applications, Academic Press, 2020.

[34] K. Shafique, B. A. Khawaja, F. Sabir, S. Qazi, and M. Mustaqim, "Internet of Things (IoT) for next-gneration smart systems: a review of current challenges, future trends and prospects for emerging 5G-IoT scenarios," IEEE Access, vol. 8, pp. 2302223040, 2020.

[35] Q. Chen, K. Yang, H. Jiang, and M. Qiu, "Joint beamforming coordination and user selection for CoMP enabled NR-U networks," IEEE Internet of Things Journal, 2021.

[36] M. B. Hassan, S. Alsharif, H. Alhumyani, E. S. Ali, R. A. Mokhtar, and R. A. Saeed, "An enhanced cooperative communication scheme for physical uplink shared channel in NB-IoT," Wireless Personal Communications, vol. 120, no. 3, pp. 23672386, 2021.

[37] A. Taufique, M. Jaber, A. Imran, Z. Dawy, and E. Yacoub, "Planning wireless cellular networks of future: outlook, challenges and opportunities," IEEE Access, vol. 5, pp. 48214845, 2017.

[38] K. Nuanyai and S. Chantaraskul, “Actual Traffic Based LoadAware Dynamic Point Selection for LTE-Advanced System, Advances in Science," Technology and Engineering Systems Journal, vol. 6, no. 2, pp. 776-783, 2021. 